\title{
ON A CERTAIN LUBRICATION SLIP MODEL
}

\author{
KHALID AIT HADI AND MY HAFID EL BANSAMI
}

Received 2 May 2005 and in revised form 20 September 2005

We consider a stationary equation modeling the slip flow in a hard disk drive. A result of existence is proven under different hypotheses than in the literature. The uniqueness of solution followed from monotonicity techniques for nonlinear PDEs.

\section{Introduction}

The Ky Fan's inequality is one of the most important results in nonlinear analysis. Indeed, many practical and theoretical results, from various fields, are derived from it. Therefore, its application attracted many researchers (see $[2,3])$ and considerable results have been obtained in this field of research. In this paper, we apply the theory related to the Ky Fan's inequality to treat the second-order slip equation modeling the performance of the air bearing operating system.

The normalized second-order slip Reynolds equation considered in this paper is the following (see $[8,9])$ :

$$
\nabla\left[\left(H^{3} P+6 K H^{2}+6 K^{2} \frac{H}{P}\right) \nabla P\right]=\Lambda \cdot \nabla(P H)
$$

where the air bearing normalized pressure $P=P(x)$ is the unknown of the problem, the normalized film thickness between the head and the magnetic disk is given by $H=H(x)$, $K>0$ is the so-called Knudsen number, $\Lambda$ is the bearing vector, and $\Omega \subseteq \mathbb{R}^{2}$ is the region (with smooth boundary $\partial \Omega$ ) where the upper and lower bodies are in proximity.

In [4], Chipot and Luskin studied an analogous equation without the $6 K^{2}(H / P)$ term, they proved existence and uniqueness by using a change of the unknown function which leads to a new problem in which the nonlinearity appears in the convection term and they used directly a fixed point argument to the problem. Due to the $6 K^{2}(H / P)$ term, this proof does not work in our case to prove existence (see Remark 3.3), which motivated us to look for applying other techniques than those of the fixed point. This has been made in our work [1] where the existence of solutions was proved under some conditions on the data of the problem. In this paper, we continue our investigation concerning the same 
problem, we prove an existence result with different hypotheses on the data, and we also prove the uniqueness of solution using some monotonicity arguments.

\section{Existence of solutions}

We consider the following problem $(\mathscr{P})$

$$
(\mathscr{P}) \begin{cases}\nabla\left[\left(H^{3} P+6 K H^{2}+6 K^{2} \frac{H}{P}\right) \nabla P\right]=\Lambda \cdot \nabla(P H) & \text { in } \Omega, \\ P=\Psi & \text { in } \partial \Omega .\end{cases}
$$

We assume that the functions $H: \Omega \rightarrow \mathbb{R}$ and $\Psi: \partial \Omega \rightarrow \mathbb{R}$ satisfy the following hypotheses:

$$
H \in W^{1, \infty}(\Omega),
$$

$H$ is bounded in $W^{1, \infty}(\Omega), \quad 0<a \leq H(x) \leq b \quad$ a.e. in $\Omega$.

$\Psi$ is the restriction to $\partial \Omega$ of a smooth function $\widetilde{\Psi}$ defined on $\Omega$, such that

$$
\|\nabla \tilde{\Psi}\|_{L^{2}(\Omega)} \leq M
$$

In order to give a variational formulation of $(\mathscr{P})$, we introduce the following set:

$$
V:=\left\{u \in H^{1}(\Omega) \cap L^{\infty}(\Omega) \mid \exists \alpha>0 \text { such that } u(x) \geq \alpha \text { a.e. in } \Omega\right\} .
$$

In the following, we will use the notation

$$
B_{r}:=\left\{u \in H^{1}(\Omega) \mid u(x) \geq r \text { a.e. in } \Omega\right\} .
$$

Throughout this paper, $\|\cdot\|$ denotes the norm in $L^{2}(\Omega)$.

Definition 2.1. We say that $P$ is a weak solution of $(\mathscr{P})$ if $P-\widetilde{\Psi} \in H_{0}^{1}(\Omega), P \in V$, and

$$
\int_{\Omega}\left(H^{3} P+6 K H^{2}+6 K^{2} \frac{H}{P}\right) \nabla P \cdot \nabla v d x=\int_{\Omega} P H \Lambda \cdot \nabla v d x, \quad \forall v \in H_{0}^{1}(\Omega) .
$$

In order to prove the existence, we need the following technical estimation; first we consider the following inequality:

$$
\int_{\Omega}\left(H^{3} P+6 K H^{2}+6 K^{2} \frac{H}{P}\right) \nabla P \cdot \nabla(P-\tilde{\Psi}) d x \leq \int_{\Omega} P H \Lambda \cdot \nabla(P-\tilde{\Psi}) d x
$$

Proposition 2.2. If $P$ is a solution of (2.7) such that $P \in B_{r_{1}}$ and $r_{1}$ satisfies the following hypothesis:

$$
r_{1} a^{3}+6 K a^{2}>C_{p}|\Lambda|+C_{p} b^{3}\|\nabla \tilde{\Psi}\|
$$

(where $C_{p}$ is the constant of Poincaré [7] and $|\Lambda|$ is the Euclidean norm of $\Lambda$ ), then

$$
\|\nabla P\| \leq C
$$


Proof. We put $z=P-\tilde{\Psi} \in H_{0}^{1}(\Omega)$, the inequality (2.7) can be written as

$$
\begin{gathered}
\int_{\Omega}\left(H^{3} P+6 K H^{2}+6 K^{2} \frac{H}{P}\right) \nabla(z+\tilde{\Psi}) \cdot \nabla z d x \leq \int_{\Omega}(z+\tilde{\Psi}) H \Lambda \cdot \nabla z d x \\
\int_{\Omega}\left(H^{3} P+6 K H^{2}\right) \nabla z^{2} d x+\int_{\Omega} 6 K^{2} \frac{H}{P} \nabla z^{2} d x \\
\leq \int_{\Omega} H \Lambda \cdot z \nabla z d x+\int_{\Omega} H \Lambda \cdot \tilde{\Psi} \nabla z d x-\int_{\Omega} H^{3} P \nabla \tilde{\Psi} \cdot \nabla z d x \\
-\int_{\Omega} 6 K H^{2} \nabla \tilde{\Psi} \cdot \nabla z d x-\int_{\Omega} 6 K^{2} \frac{H}{P} \nabla \tilde{\Psi} \cdot \nabla z d x
\end{gathered}
$$

then

$$
\begin{aligned}
\left(a^{3} r_{1}+6 K a^{2}\right)\|\nabla z\|^{2} \leq & C_{p}|\Lambda| b\|\nabla z\|^{2}+|\Lambda| b\|\tilde{\Psi}\|\|\nabla z\| \\
& +C_{p} b^{3}\|\nabla \tilde{\Psi}\|\|\nabla z\|^{2}+b^{3}\|\tilde{\Psi}\|\|\nabla \tilde{\Psi}\|\|\nabla z\| \\
& +6 K b^{2}\|\nabla \tilde{\Psi}\|\|\nabla z\|+6 K^{2} \frac{b}{r_{1}}\|\nabla \tilde{\Psi}\|\|\nabla z\|
\end{aligned}
$$

that is,

$$
\begin{aligned}
\left(a^{3} r_{1}\right. & \left.+6 K a^{2}-C_{p}|\Lambda| b-b^{3}\|\nabla \tilde{\Psi}\|\right)\|\nabla z\| \\
& \leq|\Lambda| b\|\tilde{\Psi}\|+b^{3}\|\tilde{\Psi}\|\|\nabla \tilde{\Psi}\|+6 K b^{2}\|\nabla \tilde{\Psi}\|+6 K^{2} \frac{b}{r_{1}}\|\nabla \tilde{\Psi}\|
\end{aligned}
$$

However, if the hypothesis (2.8) is verified, therefore $\|\nabla z\| \leq$ Cte, with Cte $=(|\Lambda| b\|\tilde{\Psi}\|+$ $\left.b^{3}\|\tilde{\Psi}\|\|\nabla \tilde{\Psi}\|+6 K b^{2}\|\nabla \tilde{\Psi}\|+6 K^{2}\left(b / r_{1}\right)\|\nabla \tilde{\Psi}\|\right) /\left(a^{3} r_{1}+6 K a^{2}-C_{p}|\Lambda| b-b^{3}\|\nabla \tilde{\Psi}\|\right)$. It follows that

$$
\|\nabla P\| \leq\|\nabla z\|+\|\nabla \tilde{\Psi}\| \leq C .
$$

Remark 2.3. Independently of data, we can always find $r_{1}$ such that

$$
r_{1}>\frac{C_{p}|\Lambda|+C_{p} b^{3}\|\nabla \tilde{\Psi}\|-6 K a^{2}}{a^{3}} .
$$

We prove the existence of a weak solution of $(\mathscr{P})$ by using a change of the unknown function [1]. Let us write for $P>0$ that

$$
\begin{aligned}
\left(H^{3} P+6 K H^{2}+6 K^{2} \frac{H}{P}\right) \nabla P= & H^{3} \nabla\left(\frac{P^{2}}{2}+6 K \frac{P}{H}+6 K^{2} \frac{\log (P)}{H^{2}}\right) \\
& +6 K P H \nabla H+12 K^{2} \log (P) \nabla H .
\end{aligned}
$$


The new unknown function will be

$$
u=\frac{P^{2}}{2}+6 K \frac{P}{H}+6 K^{2} \frac{\log (P)}{H^{2}}
$$

We consider the function $g:] 0,+\infty[\rightarrow \mathbb{R}$,

$$
g(t)=\frac{t^{2}}{2}+6 K t+6 K^{2} \log (t)
$$

It is easy to see that $g$ is an increasing and bijective function. We deduce from the above definition that

$$
P=\frac{1}{H} \kappa(x, u)
$$

with

$$
\kappa(x, u)=g^{-1}\left(H^{2} u+6 K^{2} \log H\right) .
$$

Our initial problem $(\mathscr{P})$ becomes in $u$

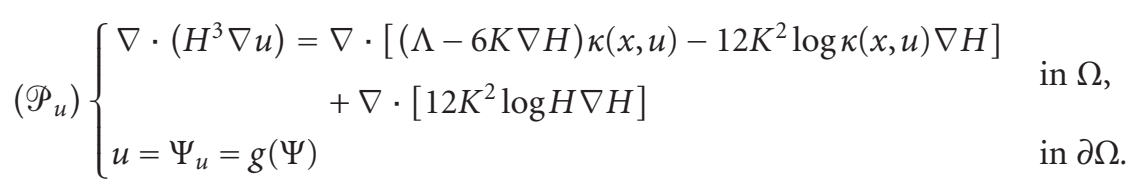

We set $\tilde{\Psi}_{u}=g(\tilde{\Psi})$.

Definition 2.4. $u$ is a weak solution of $\left(\mathscr{P}_{u}\right)$ if $u-\widetilde{\Psi}_{u} \in H_{0}^{1}(\Omega)$ and

$$
\begin{array}{r}
\int_{\Omega} H^{3} \nabla u \cdot \nabla v d x=\int_{\Omega}\left[(\Lambda-6 K \nabla H) \kappa(x, u)-12 K^{2} \log \kappa(x, u) \nabla H\right. \\
\left.+12 K^{2} \log H \nabla H\right] \cdot \nabla v d x, \quad \forall v \in H_{0}^{1}(\Omega) .
\end{array}
$$

The equivalence between $(\mathscr{P})$ and $\left(\mathscr{P}_{u}\right)$ is given by the following lemma.

LEMMA 2.5. $u$ is a weak solution of $\left(\mathscr{P}_{u}\right)$ if and only if $P$, given by (2.18), is a weak solution of $(\mathscr{P})$.

Proof. It is clear from (2.15) that the two variational formulas are equivalent. And from (2.16), it is obvious that if $P \in V$, then $u \in H^{1}(\Omega)$. It remains to show that if $u$ is a solution of $\left(\mathscr{P}_{u}\right)$, then $P \in V$. From (2.18), we have that $P \in H^{1}(\Omega)$ since $\left(g^{-1}\right)^{\prime}$ is bounded. On the other hand, we have classically $u \in L^{\infty}(\Omega)$. From (2.18), we deduce that $P$ belongs to $L^{\infty}(\Omega)$ with $P$ bounded away from 0 , and the proof is ended. 
We consider the following two inequalities:

$$
\begin{gathered}
\int_{\Omega}\left(H^{3} P+6 K H^{2}+6 K^{2} \frac{H}{P}\right) \nabla P \cdot \nabla(P-\tilde{\Psi}) d x \leq \int_{\Omega} P H \Lambda \cdot \nabla(P-\tilde{\Psi}) d x \\
\int_{\Omega} H^{3} \nabla w \cdot \nabla w d x \leq \int_{\Omega}(\Lambda-6 K \nabla H) \kappa_{1}(x, w) \cdot \nabla w d x-\int_{\Omega} 12 K^{2} \log \kappa_{1}(x, w) \nabla H \cdot \nabla w d x \\
+\int_{\Omega} 12 K^{2} \log H \nabla H \cdot \nabla w d x-\int_{\Omega} H^{3} \nabla \tilde{\Psi}_{u} \cdot \nabla w d x
\end{gathered}
$$

with $\kappa_{1}$ such that $\kappa_{1}(x, \tilde{w})=\kappa\left(x, \tilde{w}+\tilde{\Psi}_{u}\right)$ and $w=u-\tilde{\Psi}_{u}$.

Due to the fact that for all $s \in \mathbb{R}$,

$$
\begin{gathered}
0 \leq \frac{d g^{-1}}{d s}(s)=\frac{g^{-1}(s)}{\left(g^{-1}\right)^{2}(s)+6 K g^{-1}(s)+6 K^{2}} \leq \frac{1}{6 K}, \\
0 \leq \frac{d}{d s} \log \left(g^{-1}(s)\right)=\frac{1}{\left(g^{-1}\right)^{2}(s)+6 K g^{-1}(s)+6 K^{2}} \leq \frac{1}{6 K^{2}},
\end{gathered}
$$

we have the following result.

LEMMA 2.6. Let $w$ be a solution of the inequality (2.23) such that $w+\widetilde{\Psi}_{u} \geq r_{2}$ with $r_{2}$ verifies the condition

$$
r_{2} \geq \frac{g\left(r_{1} b\right)-6 K^{2} \log (a)}{a^{2}}
$$

then $\|\nabla w\| \leq C_{1}$ with $C_{1}$ a positive constant.

Proof. Let $w$ be a solution of (2.23), we make the inverse change of variable, we deduce that $P$ verifies (2.22). Since $r_{2}$ satisfies (2.25), then $P \in B_{r_{1}}$ with $r_{1}$ verifies the condition (2.8). And according to Proposition 2.2, there exists a constant $C$ such that $\|\nabla P\| \leq C$, while using (2.18), (2.19), and (2.24), we get $\|\nabla w\| \leq C_{1}$.

Now we give the main result of this section.

Theorem 2.7. If

$$
(H)\left\{\begin{array}{l}
\widetilde{\Psi}_{u} \geq \frac{g\left(r_{1} b\right)-6 K^{2} \log (a)}{a^{2}} \quad \text { a.e. in } \Omega, \\
\text { with } r_{1} \text { verifies the hypothesis }(2.8),
\end{array}\right.
$$

then there exists at least one weak solution for $(\mathscr{P})$.

For the proof, we need the following proposition which is a generalization of the Ky Fan's lemma.

Notation 2.8. We denote by $\mathscr{F}(X)$ the family of all nonempty finite subsets of $X$ and by $\mathscr{F}\left(X, x_{0}\right)$ all elements of $\mathscr{F}(X)$ containing $x_{0}$. We will denote by $\operatorname{conv}(A)$ the convex hull of $A$, by $\bar{A}^{X}$ the closure of $A$ in $X$, and by $\operatorname{int}_{X}(A)$ the interior of $A$ in $X$. 
Definition 2.9 [6]. $\Sigma: X \rightarrow 2^{E}$ is said to be a KKM-application if for all $A \in \mathscr{F}(X), \operatorname{conv}(A) \subseteq$ $\bigcup_{\chi \in A} \Sigma(\chi)$.

Proposition 2.10 [5]. Let $X$ be a nonempty convex subset $\subseteq E$ (a topological vector space) and $\Sigma: X \rightarrow 2^{E}$ is a KKM-application, suppose that there exists $x_{0} \in X$ such that

(i) $\overline{\sum\left(x_{0}\right) \cap X} X^{X}$ is compact on $X$;

(ii) for all $A \in \mathscr{F}\left(X, x_{0}\right)$, for all $\chi \in \operatorname{conv}(A), \Sigma(\chi) \cap \operatorname{conv}(A)$ is closed in $\operatorname{conv}(A)$;

(iii) for all $A \in \mathscr{F}\left(X, x_{0}\right), \overline{X \cap\left(\bigcap_{\chi \in \operatorname{conv}(A)} \Sigma(\chi)\right)} X \cap \operatorname{conv}(A)=\left(\bigcap_{\chi \in \operatorname{conv}(A)} \Sigma(\chi)\right) \cap \operatorname{conv}(A)$. Then $\bigcap_{\chi \in X} \Sigma(\chi) \neq \varnothing$.

First, we will prove the following lemma which will be useful thereafter.

Lemma 2.11. The following application

$$
\begin{gathered}
\Pi(\chi, \cdot): H_{0}^{1}(\Omega) \longrightarrow \mathbb{R}, \\
q \longmapsto \int_{\Omega} H^{3} \nabla q \cdot \nabla(q-\chi) d x-\int_{\Omega} F(q) \cdot \nabla(q-\chi) d x,
\end{gathered}
$$

with

$$
F(q):=(\Lambda-6 K \nabla H) \kappa_{1}(x, q)-12 K^{2} \log \kappa_{1}(x, q) \nabla H+12 K^{2} \log H \nabla H-H^{3} \nabla \tilde{\Psi}_{u}
$$

is weakly lower semicontinuous.

Proof. Let $q_{n}$ weakly converges to $q$ in $H_{0}^{1}(\Omega)$, then there exists a subsequence $q_{n_{k}}$ such that $q_{n_{k}}$ tends to $q$ in $L^{2}(\Omega)$ and $\nabla q_{n_{k}}$ weakly converges to $\nabla q$ in $L^{2}(\Omega)$, therefore while using Lebesgue's dominated convergence theorem and estimation (2.24), we obtain

$$
\begin{aligned}
\int_{\Omega} a_{2} F\left(q_{n_{k}}\right) \cdot \nabla\left(q_{n_{k}}-\chi\right) d x & =\int_{\Omega} a_{2} F\left(q_{n_{k}}\right) \cdot \nabla q_{n_{k}} d x-\int_{\Omega} a_{2} F\left(q_{n_{k}}\right) \cdot \nabla \chi d x \\
& \longrightarrow \int_{\Omega} a_{2} F(q) \cdot \nabla q d x-\int_{\Omega} a_{2} F(q) \cdot \nabla \chi d x .
\end{aligned}
$$

For the other term of $\Pi\left(\chi, q_{n_{k}}\right)$, we have

$$
\int_{\Omega} H^{3} \nabla q_{n_{k}} \cdot \nabla\left(q_{n_{k}}-\chi\right) d x=\int_{\Omega} H^{3} \nabla q_{n_{k}} \cdot \nabla q_{n_{k}} d x-\int_{\Omega} H^{3} \nabla q_{n_{k}} \cdot \nabla \chi d x .
$$

As $\nabla q_{n_{k}}$ weakly converges to $\nabla q$ in $L^{2}(\Omega)$, then $\int_{\Omega} H^{3} \nabla q_{n_{k}} \cdot \nabla \chi d x$ converges to $\int_{\Omega} H^{3} \nabla q$. $\nabla \chi d x$. Moreover, as the application $T: L^{2}(\Omega) \rightarrow \mathbb{R}, z \mapsto \int_{\Omega} H^{3} z^{2} d x$ is convex and weakly semicontinuous in $L^{2}(\Omega)$, then $\underline{\lim }\left(H^{3}\left(\left(\nabla q_{n k}\right)^{2}-(\nabla q)^{2}\right)\right) \geq 0$. From where the result follows.

Proof of Theorem 2.7. According to Lemma 2.5, it is sufficient to prove the existence of a solution for the following problem. 
Find $w \in H_{0}^{1}(\Omega)$ such that

$$
\begin{aligned}
\int_{\Omega} H^{3} \nabla w \cdot \nabla v d x= & \int_{\Omega}(\Lambda-6 K \nabla H) \kappa_{1}(x, w) \cdot \nabla v d x-\int_{\Omega} 12 K^{2} \log \kappa_{1}(x, w) \nabla H \cdot \nabla v d x \\
& +\int_{\Omega} 12 K^{2} \log H \nabla H \cdot \nabla v d x-\int_{\Omega} H^{3} \nabla \tilde{\Psi}_{u} \cdot \nabla v d x \quad \forall v \in H_{0}^{1}(\Omega),
\end{aligned}
$$

with $\kappa_{1}(x, w)=\kappa\left(x, w+\widetilde{\Psi}_{u}\right)$.

Let us consider the space $E:=H_{0}^{1}(\Omega)$ endowed of its weak topology and

$$
X:=\left\{\varphi \in E \mid\|\nabla \varphi\| \leq C_{1}+\gamma, \varphi+\widetilde{\Psi}_{u} \geq r_{2} \text { a.e. in } \Omega\right\},
$$

with $\gamma$ a constant sufficiently large, $r_{2}$ satisfies $\tilde{\Psi}_{u} \geq r_{2} \geq\left(g\left(r_{1} b\right)-6 K^{2} \log (a)\right) /\left(a^{2}\right)$ a.e. in $\Omega$, and $C_{1}$ is the constant given in Lemma 2.6.

Let us consider, for every $\chi \in X$, the set

$$
\Sigma(\chi):=\left\{q \in X: \int_{\Omega} H^{3} \nabla q \cdot \nabla(q-\chi) d x-\int_{\Omega} F(q) \cdot \nabla(q-\chi) d x \leq 0\right\} .
$$

It is obvious that $q$ satisfies (2.31) if and only if $q \in \Sigma(\chi)$ for each $\chi \in E$. So, the proof of the existence of solutions is thus reduced to prove that

$$
\bigcap_{\chi \in E} \Sigma(\chi) \neq \varnothing
$$

We will show that conditions of Proposition 2.10 are satisfied.

Since the application $\chi \rightarrow \Pi(\chi, q)$ is linear, then $\Sigma$ is a KKM-application.

For condition (i), it is sufficient to take

$$
K:=X=\left\{\varphi \in E \mid\|\nabla \varphi\| \leq C_{1}+\gamma, \varphi+\widetilde{\Psi}_{u} \geq r_{2} \text { a.e. in } \Omega\right\} .
$$

From Lemma 2.11, we have that $q \mapsto \Pi(\chi, q)$ is weakly lower semicontinuous in $H_{0}^{1}(\Omega)$, so conditions (ii) and (iii) are satisfied.

It follows by application of Proposition 2.10 that there exists $w \in K$ such that

$$
\Pi(\chi, w) \leq 0 \quad \forall \chi \in X .
$$

In particular, for $\chi=0 \in X$ (due to $(H)$ ), we get that $w$ satisfies the inequality (2.23), which implies, according to Lemma 2.6, that $\|\nabla w\| \leq C_{1}$, and by following $w+\epsilon \sigma \in$ $\operatorname{int}(X)$ for all $\sigma$ in $\mathscr{D}^{+}(\Omega)$ and $\epsilon$ appropriately chosen.

Now, we put in (2.36) $\chi=w+\epsilon \sigma+\gamma \xi \in X$ for any $\xi \in H_{0}^{1}(\Omega)$ and convenient value of $\gamma$, then we get

$$
\int_{\Omega} H^{3} \nabla w \cdot \nabla(-(\epsilon \sigma+\gamma \xi)) d x-\int_{\Omega} F(w) \cdot \nabla(-(\epsilon \sigma+\gamma \xi)) d x \leq 0 \quad \forall \xi \in H_{0}^{1}(\Omega),
$$

and if we take $\xi=(1 / \gamma)(-\epsilon \sigma+\phi)$ with $\phi \in H_{0}^{1}(\Omega)$, we deduce that $w$ verifies $(2.31)$, and the proof is ended.

By following, we have solutions for the problems $\left(\mathscr{P}_{u}\right)$ and $(\mathscr{P})$. 
3232 On a certain lubrication slip model

\section{Uniqueness of solutions}

Next, we give a uniqueness result for the problem $(\mathscr{P})$ using a general monotonicity for a class of semilinear elliptic problems.

First, we prove a uniqueness and monotonicity result for weak solutions to the prob$\operatorname{lem}\left(\mathscr{P}_{u}\right)$.

Lemma 3.1. There exists uniqueness among all weak solutions to problem $\left(\mathscr{P}_{u}\right)$. Further, suppose that $u_{i}$ is a weak solution to $\left(\mathscr{P}_{u}\right)$ corresponding to the boundary data $\Psi_{u}^{i}, i=1,2$. If $\Psi_{u}^{1} \geq \Psi_{u}^{2}$ a.e. on $\partial \Omega$, then $u_{1} \geq u_{2}$ a.e. in $\Omega$.

Proof. We assume that $\Psi_{u}^{1} \geq \Psi_{u}^{2}$ a.e. on $\partial \Omega$, and we put

$$
l(x, u)=(\Lambda-6 K \nabla H) \kappa(x, u)-12 K^{2} \log \kappa(x, u) \nabla H+12 K^{2} \log H \nabla H .
$$

First, we prove that for all $\xi \in C^{\infty}(\bar{\Omega})$ and $\xi>0$, we have

$$
\int_{\left[u_{2}-u_{1}>0\right]} H^{3}(x) \nabla\left(u_{2}-u_{1}\right) \cdot \nabla \xi-\left(l\left(x, u_{2}\right)-l\left(x, u_{1}\right)\right) \cdot \nabla \xi d x \leq 0,
$$

where

$$
\left[u_{2}-u_{1}>0\right]=\left\{x \in \Omega \mid u_{2}(x)-u_{1}(x)>0\right\} .
$$

So, we consider for $\epsilon>0$ that

$$
\zeta=\min \left(\frac{\left(u_{2}-u_{1}\right)^{+}}{\epsilon}, \xi\right)
$$

where

$$
\varphi^{+}(x)=\max (\varphi(x), 0)
$$

Note that $\zeta \in H_{0}^{1}(\Omega)$ since $\zeta(x)=0$ for $x \in \partial \Omega$.

It follows from subtracting (2.21) with $u=u_{2}$ from (2.21) with $u=u_{1}$ that

$$
\int_{\Omega} H^{3}(x) \nabla\left(u_{2}-u_{1}\right) \cdot \nabla \zeta-\left(l\left(x, u_{2}\right)-l\left(x, u_{1}\right)\right) \cdot \nabla \zeta d x=0
$$

which for $\zeta$ given by (3.4) is equivalent to

$$
\begin{aligned}
& \int_{\left[u_{2}-u_{1}>\epsilon \xi\right]} H^{3}(x) \nabla\left(u_{2}-u_{1}\right) \cdot \nabla \xi-\left(l\left(x, u_{2}\right)-l\left(x, u_{1}\right)\right) \cdot \nabla \xi d x \\
& +\frac{1}{\epsilon} \int_{\left[0<u_{2}-u_{1} \leq \epsilon \xi\right]} H^{3}(x)\left|\nabla\left(u_{2}-u_{1}\right)\right|^{2} d x \\
& -\frac{1}{\epsilon} \int_{\left[0<u_{2}-u_{1} \leq \epsilon \xi\right]}\left(l\left(x, u_{2}\right)-l\left(x, u_{1}\right)\right) \cdot \nabla\left(u_{2}-u_{1}\right) d x=0,
\end{aligned}
$$


where

$$
\begin{aligned}
{\left[u_{2}-u_{1}>\epsilon \xi\right] } & =\left\{x \in \Omega \mid u_{2}(x)-u_{1}(x)>\epsilon \xi\right\}, \\
{\left[0<u_{2}-u_{1} \leq \epsilon \xi\right] } & =\left\{x \in \Omega \mid 0<u_{2}(x)-u_{1}(x) \leq \epsilon \xi\right\},
\end{aligned}
$$

we estimate the last integral above by

$$
\begin{aligned}
I= & \int_{\left[0<u_{2}-u_{1} \leq \epsilon \xi\right]}\left(l\left(x, u_{2}\right)-l\left(x, u_{1}\right)\right) \cdot \nabla\left(u_{2}-u_{1}\right) d x \\
\leq & \left(\int_{\left[0<u_{2}-u_{1} \leq \epsilon \xi\right]} H^{-3}(x)\left|l\left(x, u_{2}\right)-l\left(x, u_{1}\right)\right|^{2} d x\right)^{1 / 2} \\
& \times\left(\int_{\left[0<u_{2}-u_{1} \leq \epsilon \xi\right]} H^{3}(x)\left|\nabla\left(u_{2}-u_{1}\right)\right|^{2} d x\right)^{1 / 2} \\
\leq & \frac{1}{4} \int_{\left[0<u_{2}-u_{1} \leq \epsilon \xi\right]} H^{-3}(x)\left|l\left(x, u_{2}\right)-l\left(x, u_{1}\right)\right|^{2} d x \\
& +\int_{\left[0<u_{2}-u_{1} \leq \epsilon \xi\right]} H^{3}(x)\left|\nabla\left(u_{2}-u_{1}\right)\right|^{2} d x
\end{aligned}
$$

Due to fact that for all $s \in \mathbb{R}$,

$$
\begin{gathered}
0 \leq \frac{d g^{-1}}{d s}(s)=\frac{g^{-1}(s)}{\left(g^{-1}\right)^{2}(s)+6 K g^{-1}(s)+6 K^{2}} \leq \frac{1}{6 K}, \\
0 \leq \frac{d}{d s} \log \left(g^{-1}(s)\right)=\frac{1}{\left(g^{-1}\right)^{2}(s)+6 K g^{-1}(s)+6 K^{2}} \leq \frac{1}{6 K^{2}},
\end{gathered}
$$

and $H \in W^{1, \infty}(\Omega)$, and the inequality $\sqrt{A}+\sqrt{B} \geq \sqrt{A+B}$, it is easy to check that

$$
\exists M>0, \quad\left|l\left(x, u_{1}\right)-l\left(x, u_{2}\right)\right|^{2} \leq M\left|u_{1}-u_{2}\right|, \quad \forall x \in \Omega, u_{2} \in \mathbb{R},
$$

we have also

$$
H^{3}(x) \geq a^{3}>0 \quad \text { a.e. } x \in \Omega,
$$

using the estimate (3.9) in (3.7), we obtain from (3.11) and (3.12)

$$
\begin{aligned}
& \int_{\left[u_{2}-u_{1}>\epsilon \xi\right]} H^{3}(x) \nabla\left(u_{2}-u_{1}\right) \cdot \nabla \xi-\left(l\left(x, u_{2}\right)-l\left(x, u_{1}\right)\right) \cdot \nabla \xi d x \\
& \quad \leq \frac{1}{4 \epsilon} \int_{\left[0<u_{2}-u_{1} \leq \epsilon \xi\right]} H^{-3}(x)\left|l\left(x, u_{2}\right)-l\left(x, u_{1}\right)\right|^{2} d x \leq \frac{M \max \xi}{4 a^{3}} \int_{\left[0<u_{2}-u_{1} \leq \epsilon \xi\right]} d x .
\end{aligned}
$$

Now the measure of the set $\left[0<u_{2}-u_{1} \leq \epsilon \xi\right]$ goes to zero as $\epsilon \rightarrow 0$. Thus, the estimate (3.2) follows from (3.13).

Now, we set $V=\left(V_{1}, V_{2}\right)=\left(-\left(\Lambda_{2}-6 K \nabla_{2} H\right),\left(\Lambda_{1}-6 K \nabla_{1} H\right)\right)$, and $s>0$. We then set

$$
\xi\left(x_{1}, x_{2}\right)=W-\exp \left(s\left(V_{1} x_{1}+V_{2} x_{2}\right)\right)
$$


3234 On a certain lubrication slip model

where $W$ is a constant chosen large enough so that $\xi>0$. If we set $\xi$ from (3.14) in (3.2), we obtain

$$
\int_{\left[u_{2}-u_{1}>0\right]} H^{3}(x) \nabla\left(u_{2}-u_{1}\right) \cdot \nabla \xi+12 K^{2}\left(\log \kappa\left(x, u_{2}\right)-\log \kappa\left(x, u_{1}\right)\right) \nabla H \cdot \nabla \xi d x \leq 0,
$$

since $(\Lambda-6 K \nabla H) \cdot \nabla \xi=0$ for all $x \in \Omega$. Now it follows from integration by parts that

$$
\begin{aligned}
\int_{\left[u_{2}-u_{1}>0\right]} H^{3}(x) \nabla\left(u_{2}-u_{1}\right) \cdot \nabla \xi d x & =\int_{\Omega} H^{3}(x) \nabla\left(u_{2}-u_{1}\right)^{+} \cdot \nabla \xi d x \\
& =-\int_{\Omega}\left(u_{2}-u_{1}\right)^{+} \nabla \cdot\left(H^{3}(x) \nabla \xi\right) d x \\
& =-\int_{\left[u_{2}-u_{1}>0\right]}\left(u_{2}-u_{1}\right) \nabla \cdot\left(H^{3}(x) \nabla \xi\right) d x
\end{aligned}
$$

so from (3.15), we obtain

$$
\int_{\left[u_{2}-u_{1}>0\right]}\left(u_{2}-u_{1}\right)\left[-\nabla \cdot\left(H^{3}(x) \nabla \xi\right)+j(x) \nabla H \cdot \nabla \xi\right] d x \leq 0,
$$

where

$$
j(x)= \begin{cases}\frac{12 K^{2}\left(\log \kappa\left(x, u_{2}(x)\right)-\log \kappa\left(x, u_{1}(x)\right)\right)}{u_{2}(x)-u_{1}(x)} & \text { if } u_{2}(x) \neq u_{1}(x), \\ 0 & \text { if } u_{2}(x)=u_{1}(x),\end{cases}
$$

if $K=0$, then $j=0$. Further, for $K \neq 0$,

$$
\left|\log \kappa\left(x, u_{2}\right)-\log \kappa\left(x, u_{1}\right)\right| \leq \frac{C}{K^{2}}\left|u_{2}-u_{1}\right|
$$

Thus, $j \in L^{\infty}(\Omega)$. Also we have that

$$
\begin{aligned}
-\nabla & \cdot\left(H^{3}(x) \nabla \xi\right)+j(x) \nabla H \cdot \nabla \xi \\
& =-H^{3}(x) \triangle \xi-\nabla H^{3} \cdot \nabla \xi+j(x) \nabla H \cdot \nabla \xi \\
& =\exp \left(s\left(V_{1} x_{1}+V_{2} x_{2}\right)\right)\left[H^{3}(x) s^{2} V^{2}+\left(\nabla H^{3} \cdot V\right) s-j(x)(\nabla H \cdot V) s\right]
\end{aligned}
$$

hence, it follows that for $s$ sufficiently large,

$$
-\nabla \cdot\left(H^{3}(x) \nabla \xi\right)+j(x) \nabla H \cdot \nabla \xi>0
$$

for all $x \in \Omega$. Inequalities (3.17) and (3.21) thus allow us to conclude that $\left(u_{2}-u_{1}\right)^{+}=0$ a.e. This concludes the proof of the lemma.

Proposition 3.2. There exists uniqueness among all weak solutions to the problem (PP). Proof. The proof is an obvious consequence of Lemmas 2.5 and 3.1. 
Remark 3.3. Due to the estimation

$$
0 \leq \frac{d}{d s} \log \left(g^{-1}(s)\right)=\frac{1}{\left(g^{-1}\right)^{2}(s)+6 K g^{-1}(s)+6 K^{2}} \leq \frac{1}{6 K^{2}},
$$

the behaviour of the function $s \rightarrow \log \left(g^{-1}(s)\right)$ is linear for $s \rightarrow-\infty$. So, we cannot obtain an estimation for $\log \kappa(x, u)$ of the type $|\log \kappa(x, u)| \leq c|u|^{\tau}$ with $0<\tau<1$ which is the key in [4] for the proof of the existence. For this reason, we opted for another technique to prove the existence of solutions for $\left(\mathscr{P}_{u}\right)$.

\section{References}

[1] K. Ait Hadi and M. H. El Bansami, On the second order slip Reynolds equation with molecular dynamics: existence and uniqueness, JIPAM. J. Inequal. Pure Appl. Math. 5 (2004), no. 2, Article 50, 11.

[2] C. Baiocchi and A. Capelo, Variational and Quasivariational Inequalities. Applications to Free Boundary Problems, A Wiley-Interscience Publication, John Wiley \& Sons, New York, 1984.

[3] O. Chadli, Z. Chbani, and H. Riahi, Recession methods for equilibrium problems and applications to variational and hemivariational inequalities, Discrete Contin. Dynam. Systems 5 (1999), no. 1, 185-196.

[4] M. Chipot and M. Luskin, Existence and uniqueness of solutions to the compressible Reynolds lubrication equation, SIAM J. Math. Anal. 17 (1986), no. 6, 1390-1399.

[5] M. S. R. Chowdhury and K.-K. Tan, Generalization of Ky Fan's minimax inequality with applications to generalized variational inequalities for pseudo-monotone operators and fixed point theorems, J. Math. Anal. Appl. 204 (1996), no. 3, 910-929.

[6] A. El Arni, Generalized quasi-variational inequalities on non-compact sets with pseudo-monotone operators, J. Math. Anal. Appl. 249 (2000), no. 2, 515-526.

[7] L. C. Evans and R. F. Gariepy, Measure Theory and Fine Properties of Functions, Studies in Advanced Mathematics, CRC Press, Florida, 1992.

[8] Y. T. Hsia and G. A. Domoto, An experimental investigation of molecular rarefaction effects in gas lubricated bearings at ultra-low clearances, ASME J. Tribol. 105 (1983), no. 1, 120-130.

[9] Y. Sun, W. K. Chan, and N. Liu, A slip model with molecular dynamics, J. Micromech. Microeng. 12 (2002), 316-322.

Khalid Ait Hadi: Department of Mathematics, Faculty of Sciences-Semlalia, Cadi Ayyad University, BP 2390, Marrakech, Morocco

E-mail address: k.aithadi@ucam.ac.ma

My Hafid El Bansami: Department of Mathematics, Faculty of Sciences-Semlalia, Cadi Ayyad University, BP 2390, Marrakech, Morocco

E-mail address: h.elbansami@ucam.ac.ma 


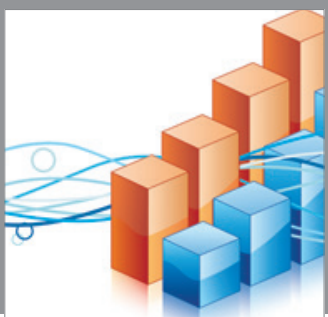

Advances in

Operations Research

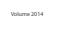

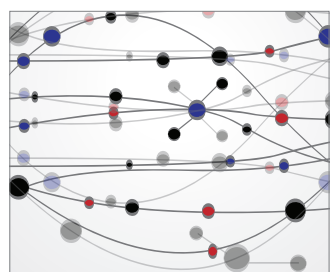

\section{The Scientific} World Journal


International Journal of

Mathematics and

Mathematical

Sciences
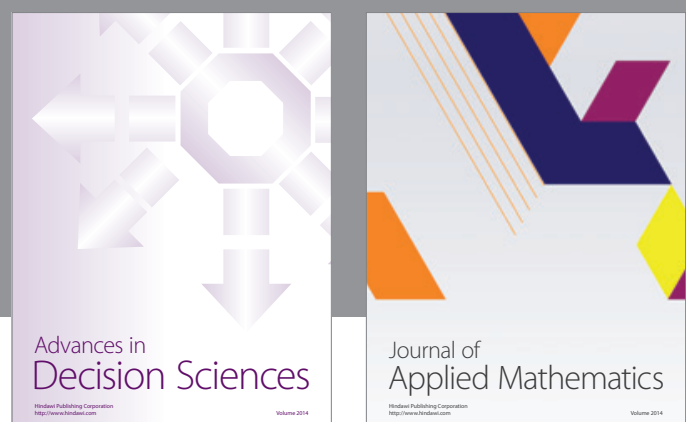

Journal of

Applied Mathematics
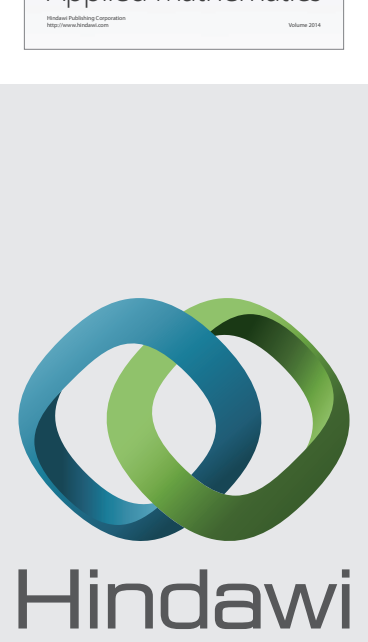

Submit your manuscripts at http://www.hindawi.com
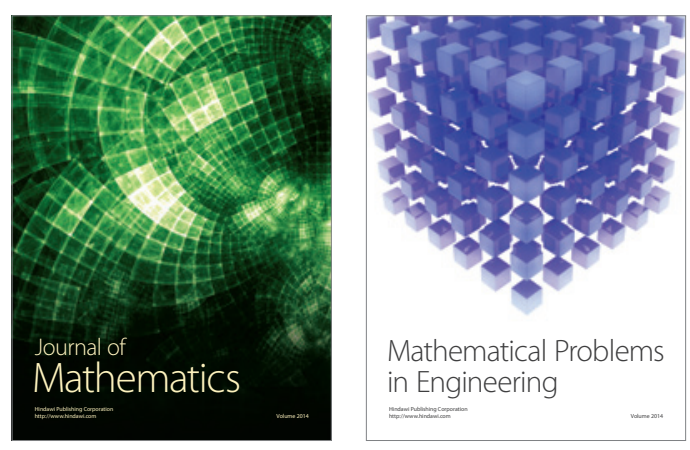

Mathematical Problems in Engineering
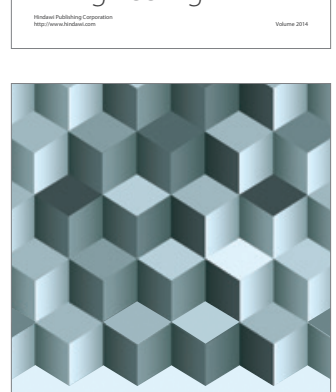

Journal of

Function Spaces
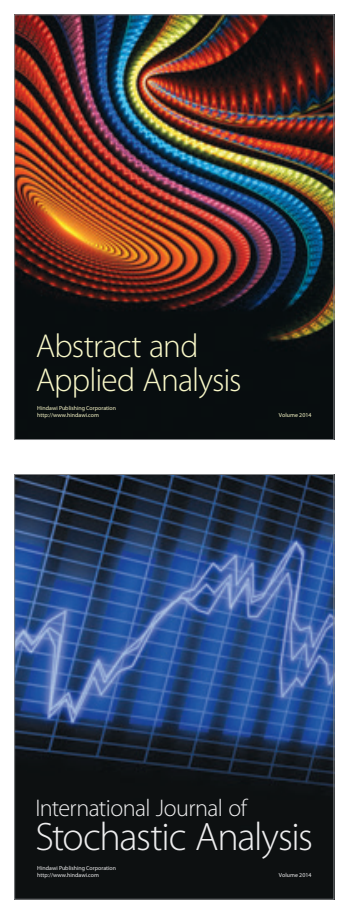

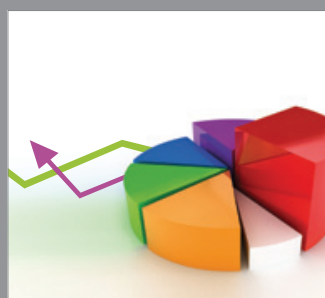

ournal of

Probability and Statistics

Promensencen
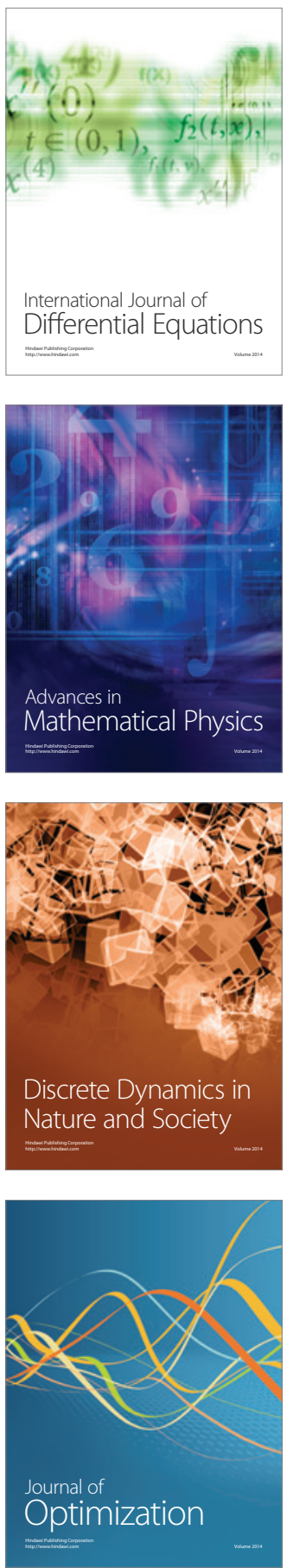